\title{
PP09
}

\section{INVESTIGATION OF LED-BASED COMPACT TRANSFER STANDARD SOURCE FOR LUMINANCE MEASUREMENT \\ Kenji Godo}

DOI 10.25039/x46.2019.PP09

from

CIE x046:2019

Proceedings

of the

29th CIE SESSION

Washington D.C., USA, June 14 - 22, 2019

(DOI 10.25039/x46.2019)

The paper has been presented at the 29th CIE Session, Washington D.C., USA, June 14-22, 2019. It has not been peer-reviewed by $\mathrm{CIE}$.

(C) CIE 2019

All rights reserved. Unless otherwise specified, no part of this publication may be reproduced or utilized in any form or by any means, electronic or mechanical, including photocopying and microfilm, without permission in writing from CIE Central Bureau at the address below. Any mention of organizations or products does not imply endorsement by the CIE.

This paper is made available open access for individual use. However, in all other cases all rights are reserved unless explicit permission is sought from and given by the CIE.

CIE Central Bureau

Babenbergerstrasse 9

A-1010 Vienna

Austria

Tel.: +4317143187

e-mail: ciecb@cie.co.at

www.cie.co.at 


\title{
INVESTIGATION OF LED-BASED COMPACT TRANSFER STANDARD SOURCE FOR LUMINANCE MEASUREMENT
}

\author{
Godo, K. \\ National Metrology Institute of Japan (NMIJ), JAPAN \\ National Institute of Advanced Industrial Science and Technology (AIST), JAPAN
}

kenji-goudo@aist.go.jp

DOI 10.25039/x46.2019.PP09

\begin{abstract}
Recently, products of light emitting diodes (LEDs) are expanding in many areas, such as lighting and display technology. As well as luminous flux measurement in the lighting technology, luminance measurement is also important in the display technology. In this study, two types of transfer standard source for LED luminance measurement, which based on a high-power LED and a homogeneous optic, was constructed according to the result of ray tracing simulation. Several evaluations were tested on those trial models, and the characteristics was tried to improve by the evaluation results. In the improved model, the luminance difference was evaluated as $\pm 2.3 \%$ within $8 \mathrm{~mm}$ in diameter or $\pm 3.2 \%$ within $10 \mathrm{~mm}$ in diameter. The luminance uniformity as a practically usable luminance source was achieved.
\end{abstract}

Keywords: Luminance, LEDs, Transfer standard, Ray tracing,

\section{Introduction}

For determining luminous flux value of lighting products, manufactures generally calibrate their instruments with a standard lamp of which reference value is traceable to National Metrology Institute. However, large discrepancy of measurement results of LEDs happened using the traditional standard lamp as a reference standard source. Therefore, NMIJ developed several transfer standard sources based on LED (standard LED) for measurement of luminous intensity and luminous flux of LED. These standard LED have suitable properties for LED measurement in manufacturers, and are used as a transfer standard source for transferring the reference value from a calibration laboratory to manufacturers in Japan.

On the other hand, as well as luminous flux measurement in lighting product, luminance measurement in display product is also indispensable. The necessity of transfer standard source for LED luminance measurement is the same situation as luminous flux measurement.

Suitable properties of both uniform luminance surface and angular distribution similar to Lambertian surface are necessary for a candidate of the luminance transfer standard source. In another studies, an integrating sphere source was investigated as the candidate of a transfer standard source. However, an integrating sphere source has some weakness at a point of portability and robustness, thus it is unsuitable for the transfer standard source. As other methods, by technological progress over the past few years, beam homogeneous technique based light source, e.g. using a microlens array or a light pipe, is becoming an attractive method for making uniform luminance surface. In the preliminary study with ray tracing simulation, we proposed new type of transfer standard source for LED luminance measurement using beam homogeneous technique.

In this study, two types of transfer standard source for LED luminance measurement (standard LED for luminance), which based on a high-power LED (HP-LED) and a homogeneous optic, has been constructed according to the result of ray tracing simulation. In addition, several evaluations were tested on those trial models, and the characteristics was tried to improve by the evaluation results. 


\section{Optical model of standard LED for luminance}

To construct the optical model of standard LED for luminance for ray tracing simulation, commercial software Zemax ${ }^{\mathrm{TM}}$ was used.

The optical model of main flame was designed as black alumite treatment surface. In the ray tracing simulations, reflectance of the black alumite surface was calculated as below $5 \%$ in visible range. From a point of portability as a transfer standard, the size of transfer standard was designed in smaller size than $60 \mathrm{~mm}$ in length and $30 \mathrm{~mm}$ in diameter, except for a light source part.

White HP-LED were selected as a light source for constructing the optical model. The spectral distributions, the spatial distributions of luminous intensity of the HP-LED were measured by the gonio-photometer system of which measurement distance is $1.2 \mathrm{~m}$. The spatial distributions are similar to Lambertian distribution. The optical properties of HP-LEDs measuring were used in ray tracing simulation of this study.

The methods for obtaining the necessary properties of standard LED for luminance, i.e. a uniform luminance surface and uniform diffused property, are described subsequently below.

\subsection{Method for making uniform luminance surface}

For creating a uniform luminance surface, two methods using microlens arrays (MLA) or light pipe (LP) was considered (MLA model and LP model), and those two methods were analysed with ray tracing simulation before construction of trial models.

MLA generally is constructed multiple lenses formed in a one-dimensional or two-dimensional array on a supporting substrate. A diameter of one lens is less than $1 \mathrm{~mm}$ and often as small as $10 \mu \mathrm{m}$. To generate a uniform luminance surface with MLA, a pair of MLAs (MLA $A_{1}$ and MLA $)$ and a condenser lens is generally used. The homogenization field is located at one focal length distance behind the condenser lens. The dimension of homogenized plane $\left(D_{F T}\right)$ is given by Eq. $(1)$,

$$
D_{F T}=\frac{P_{L A} \cdot f_{F L}}{f_{L A 1} \cdot f_{L A 2}}\left(f_{L A 1} \cdot f_{L A 2}-d_{12}\right)
$$

where

$$
\begin{array}{ll}
P_{L A} & \text { is the pitch length of pair of MLA; } \\
f_{F L} & \text { is the focal length of condenser lens; } \\
f_{L A 1}, f_{L A 2} & \text { are the focal length each of MLA; } \\
d_{12} & \text { is the distance between MLA pair. }
\end{array}
$$

$D_{F T}$ is used as a uniform luminance surface, and a surface of a precise aperture is placed in the position of $D_{F T}$.

The other method is the homogenized method using LP. LP is usually hexagonal structure, LP is much like optical fibers, that is, a homogenized method using LP utilize total internal reflection (Snell's law) to transmit light from entrance to exit of the LP. As a consequence, a uniform luminance surface is generated on the exit plane of the LP. Refractive index is the only factor that influences the LP's critical angle which defines the angle of acceptance. The homogenized method with LP does not need complicated optical calculations, but the plane size of uniform luminance surface strongly depended on size of LP.

\subsection{Method for making uniform diffused property}

The other requirement for standard LED for luminance is uniform diffused property. Ideally, diffused property of luminous intensity emitted from uniform luminance surface is preferable similar Lambertian surface. The basic feature of Lambertian surface is that luminance is a constant, independent of observation angle. Optical diffuser is often used to control diffused property or make Lambertian surface. Common diffusers, e.g. a ground glass or an opal glass, are known to be an inefficient diffuser for making Lambertian surface. 
To overcome weak points of the common diffuser, a novel approach for controlling diffused property based on arrays of randomized microlens has been developed. A microlens diffuser is constructed with generally microlens, where each individual microlens element is designed and fabricated to produce controlled diffused property. However, the arrangement in ensemble is randomized according to well-defined rules.

In this study, we tried to use the microlens diffuser mentioned above to get uniform diffused property.

Optical model of the microlens diffuser was constructed with data of Bidirectional Transmittance Distribution Function from manufacture datasheets. The manufacture datasheets do not describe surface property of the microlens diffuser, thus the optical model of microlens diffuser does not consider uniformity property of surface which affect the uniform luminance surface.

\subsection{Results of ray tracing simulation}

By some trials with the ray tracing simulation, the optical component such as MLA and LP were modeled and setting position of it was optimized.

The result of the ray tracing simulation showed that the luminance difference of MLA model achieves $\pm 4 \%$ within $5 \mathrm{~mm}$ in diameter and the angular distribution of it close to Lambertian property. On the other hand, in the LP model, it found that the uniformity area of luminance in the LP model ( $\pm 5 \%$ within $5 \mathrm{~mm}$ ) is smaller than the MLA model $( \pm 4 \%$ within $5 \mathrm{~mm})$, but the optical efficiency of the LP model is superior to the MLA model.

For more information about the ray tracing simulation, it is described in our previous study: GODO, K. Journal of Light and Visual Environment, 41.

\section{Construction of trial models and evaluation}

Figure 1 shows the schematic drawing of trial model based on MLA. The MLA model was constructed in a pair of MLAs, a condenser lens, a microlens diffuser, three baffles, and a precise aperture. Their specifications and setting positions were determined by the result of ray tracing simulation. The outer size of MLA model was designed at $65 \mathrm{~mm}$ in length and at $\phi 50$ $\mathrm{mm}$ in diameter except the LED heatsink part. The pair of MLAs was selected orthogonal lenses formed two-dimensional array on a silica substrate. In design value, the curvature radius of the orthogonal lenses was $0.4 \mathrm{~mm}$ and the pitch of neighbouring lenses is $0.2 \mathrm{~mm}$. The overall square size of the MLA is one side of $12 \mathrm{~mm}$. The precise aperture, of which opening area size is $9 \mathrm{~mm}$ in diameter, was made.

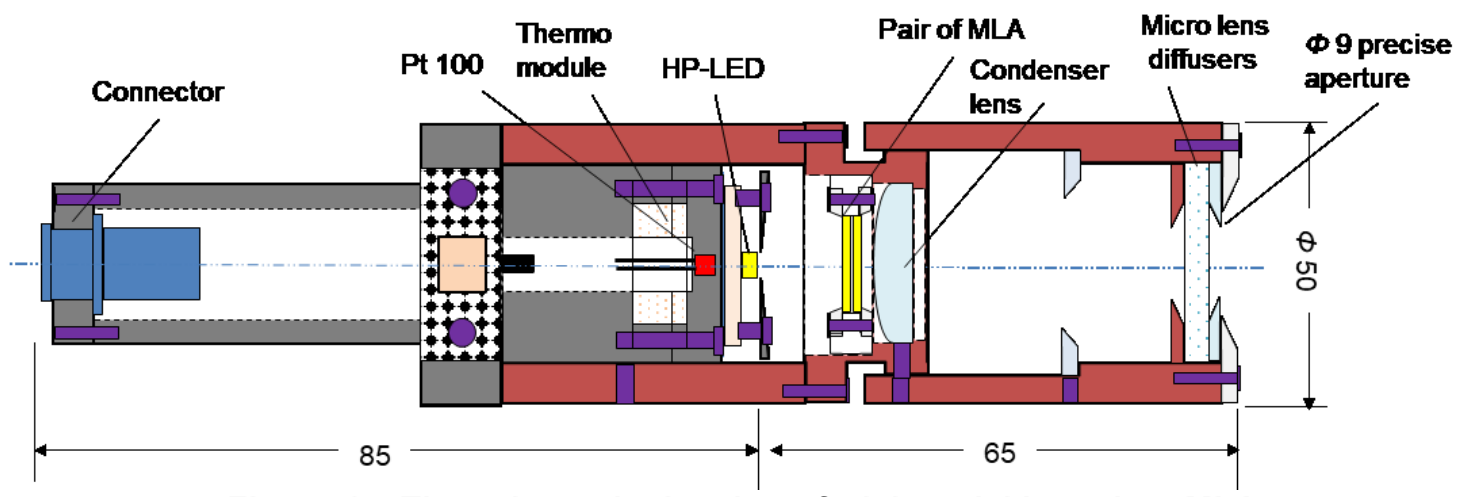

Figure 1 - The schematic drawing of trial model based on MLA

In addition, four different color high-power LED (HP-LED: Blue, Green, Red and White) were selected as a light source for constructing the LED heatsink part for the trial model. Their HPLEDs are operated at $350 \mathrm{~mA}$. Generally, temperature of HP-LEDs considerably increases during DC operation, e.g. exceeds $50{ }^{\circ} \mathrm{C}$, and long waiting time is required for stabilization of optical intensity from operating HP-LEDs. For getting stable optical intensity of HP-LED, the selected HP-LED was mounted to the temperature-stabilized heatsink in which a thermo-module and a platinum temperature sensor (Pt 100) were built. The temperature of LED heatsink was 
controlled by a commercial temperature controller. The temperature controller is specified to \pm $2 \mathrm{mK}$ temperature stability. In the preliminary measurement, the optical intensity of the HP-LED was stabilized after operating $10 \mathrm{~min}$, and the fluctuation of optical intensity did not exceed $0.1 \%$ after stabilization.

Figure 2 shows the schematic drawing of trial model based on LP. The LP model was constructed in LP, a microlens diffuser, baffles, and a precise aperture. In the LP models, the LP is used that, the length is $50 \mathrm{~mm}$, the length of between opposite side is $8 \mathrm{~mm}$, and the material of it is silica. The precise aperture, of which opening area size is $6 \mathrm{~mm}$ in diameter, was made. The LED heatsink part is the same structure as MLA model.

Figure 3 shows the evaluation results of luminance difference of the trial models. The luminance difference of each constructed models was evaluated with an image-luminance meter. The luminance difference of MA model was evaluated as $\pm 2.0 \%$ within $9 \mathrm{~mm}$ in diameter, while, the luminance difference of LP model was evaluated as $\pm 3.2 \%$ within $6 \mathrm{~mm}$ in diameter. Luminance value of the MA model with white HP-LED was averaged $3800 \mathrm{~cd} / \mathrm{m}^{2}$, and the luminance value of the LP model with same white HP-LED was averaged $40,000 \mathrm{~cd} / \mathrm{m}^{2}$. It found that the luminance uniformity area of LP model was smaller than the MLA model, but the optical efficiency of LP model was superior to the MLA model by 10 times or more. This tendency is the same as the result of the ray tracing simulation.

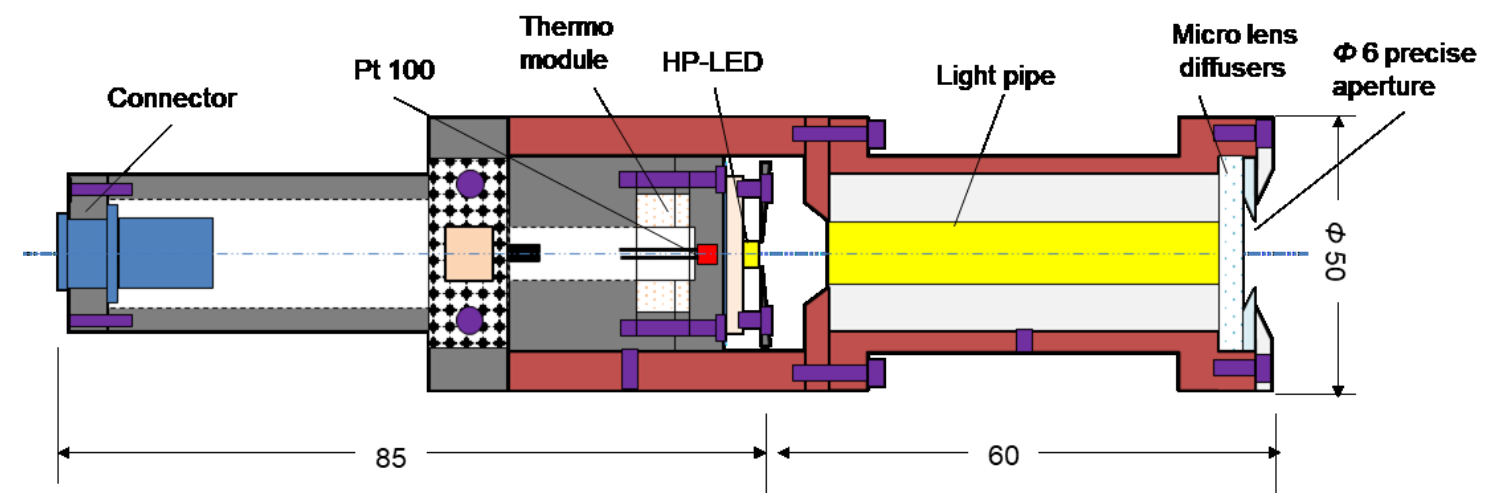

Figure 2 - The schematic drawing of trial model based on LP
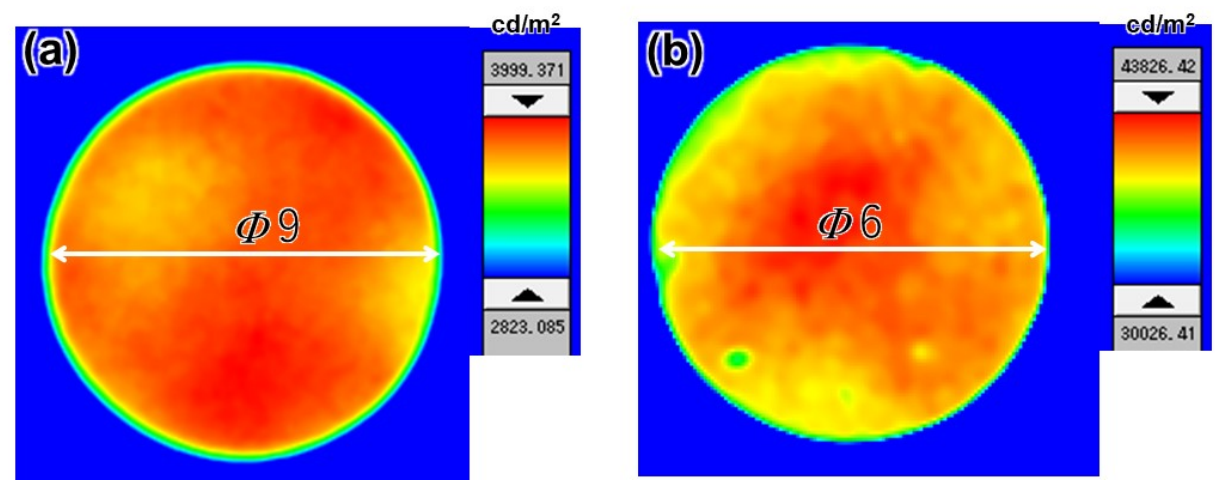

Figure 3 - Luminance difference of the trial models with white HP-LED:(a) The MLA model (averaged $3800 \mathrm{~cd} / \mathrm{m}^{2} \pm 2.0 \%$ ), (b) The LP model (averaged 40,000 cd/ $/ \mathrm{m}^{2} \pm 3.2 \%$ )

\section{Improvement of LP model}

By the evaluation results of two types trial model, the MLA model had better properties of the uniformity area of luminance, and the LP model was superior in the point of the optical efficiency. However, through multiple adjustments of optical arrangement, it was found that the uniformity area of luminance in MLA model fluctuated greatly depending on the position of microlens. We thought that it is difficult to set the distance and position of the MLA according to the optical design and the improvement of the MLA model is difficult. 
On the other hand, the area of the luminance uniformity in the LP model are depending on the LP size, and can be easily improved by increasing the LP size. The LP model has significantly better properties than the MLA model in both reproduce of optical arrangement and robustness. Thus, by increasing the size of the LP, we had developed an improved LP model that both optical efficiency and uniformity area.

Figure 4 shows the photograph of the improved LP model. Fig 4 (a) indicates overall picture, fig 4 (b) indicates the luminance surface when each color standard LED lighting, respectively. The basic structure has not changed from fig 2, and the LP size has become large. In the improved LP models, the LP was used that, the length was $75 \mathrm{~mm}$, the length of between opposite side was $12.5 \mathrm{~mm}$. The precise aperture, of which opening area size is $10.5 \mathrm{~mm}$ in diameter, was set. The outer size of improved LP model consequently was increased to $90 \mathrm{~mm}$ in length and at $44 \mathrm{~mm}$ in diameter except the LED heatsink part.

In addition, to get more optical stability, the LED heatsink part has been replaced by "HighPower type standard LED", which has already been commercialized by NICHIA corporation (GODO, K.et al., 2012). Their High-Power type standard LED are operated at $350 \mathrm{~mA}$ and their temperature are controlled at $55^{\circ} \mathrm{C}$.

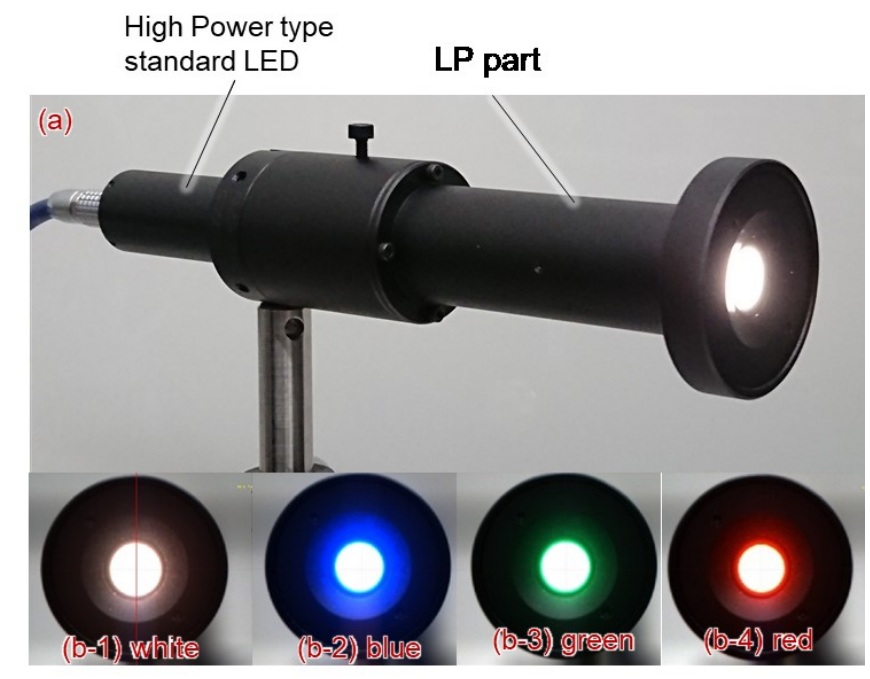

Figure 4 - Photograph of the improved LP model. (a) Overall picture, (b) Luminance surface when each High-Power type standard LED using

Figure 5 shows the luminance difference of the improved LP model evaluated by an imageluminance meter. The luminance difference is evaluated as $\pm 2.3 \%$ within $8 \mathrm{~mm}$ in diameter or $\pm 3.2 \%$ within $10 \mathrm{~mm}$ in diameter. Luminance value of the improved LP model with white HPLED is averaged $22,000 \mathrm{~cd} / \mathrm{m}^{2}$. Generally, since viewing area of a luminance meter is about 10 $\mathrm{mm}$, we think that the luminance uniformity as luminance standard source in practice has been achieved. The luminance value is reduced to about half that of the trial model, because this is a characteristic in which uniformity and intensity of luminance are contradictory. 


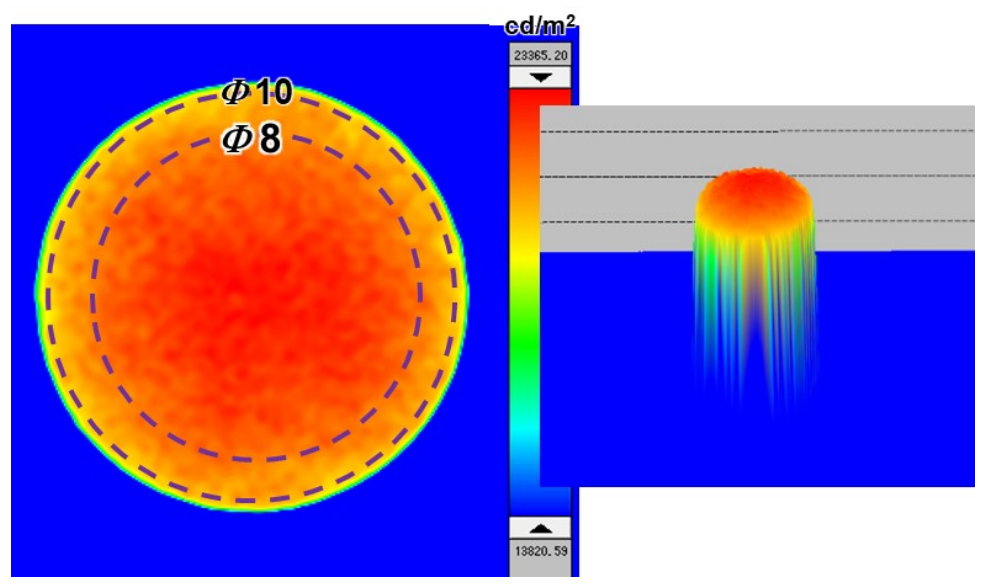

Figure 5 - Luminance uniformity of the improved LP model

The angular distribution of the luminous intensity was evaluated by a gonio-photometer system. Figure 6 shows the angular distribution of the improved LP model. The angular distributions considerably match with Lambertian distribution by using the microlens diffuser. The poor match outside the $\pm 60^{\circ}$ range is due to the knife edge angle of the precise aperture.

As a remarkable point using the microlens diffuser, luminance was more than two times stronger than when using an opal glass diffuser.

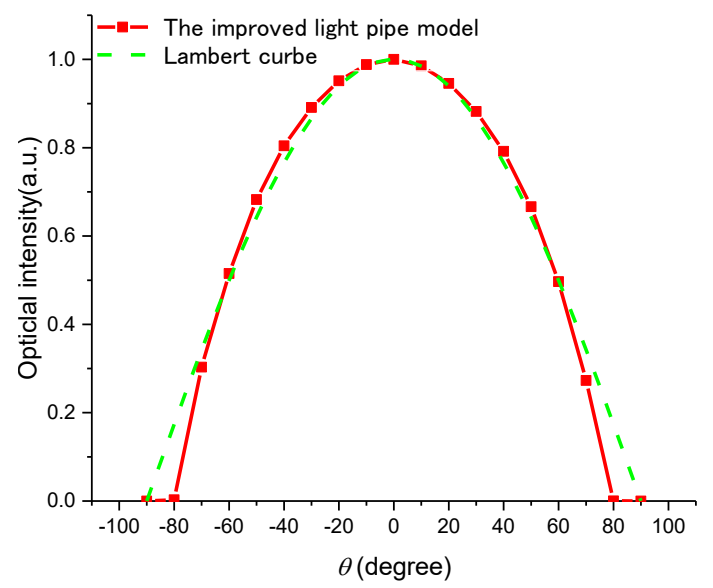

Figure 6 - Angular distribution of the improved LP model ( $\phi 0^{\circ}$ plane)

Figure 7 shows the optical stability of radiant flux of the improved LP model. The improved LP model was operated at $350 \mathrm{~mA}$ with controlled at a constant temperature of $55^{\circ} \mathrm{C}$. The optical stability was evaluated by 8 -hour operation.

This figure demonstrates that the optical stability of the improved LP model increased only $0.04 \%$ in the first 10 minutes of operation. It became almost constant quickly after 10 minutes operation. This stability is better than a typical white LED without a thermo controller. 


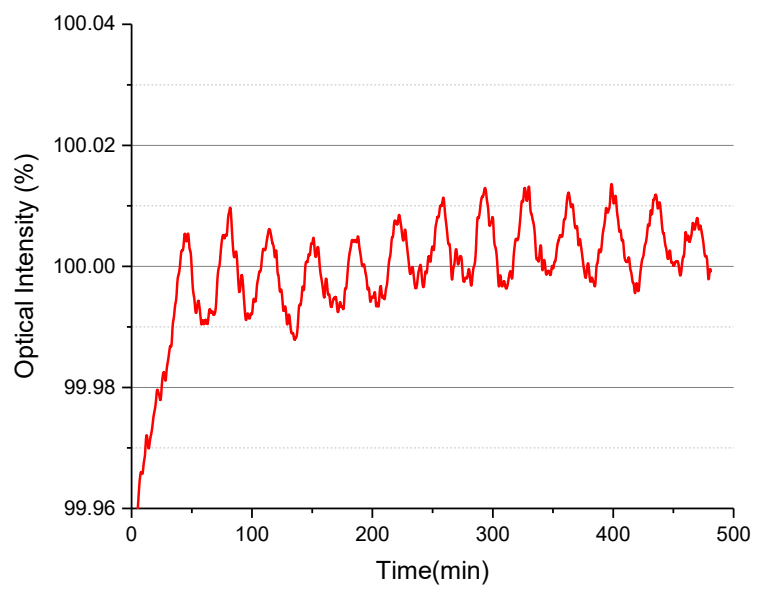

Figure 7 -Optical stability at 8-hour operation of the improved LP model

\section{Conclusion}

In this study, LED-based compact transfer standard source for luminance measurement had been developed.

Two types of standard LED for luminance which based on HP-LED and a homogeneous optic, has been constructed as a trial according to the result of ray tracing simulation. The MLA models evaluated that the luminance difference achieved $\pm 2 \%$ within $9 \mathrm{~mm}$ in diameter. On the other hand, in the LP model, it found that the uniformity area of luminance is smaller than the MLA model, but the optical efficiency of the LP model is superior to the MLA model.

The LP model is inferior in uniformity, but, the LP model has significantly better properties than the MLA model in both reproduce of optical arrangement and robustness. Therefore, we had developed an improved LP model by increasing the length of opposite side of the LP from $8 \mathrm{~mm}$ to $12.5 \mathrm{~mm}$.

The evaluation result of luminance difference of the improved LP model was evaluated as \pm $2.3 \%$ within $8 \mathrm{~mm}$ in diameter or $\pm 3.2 \%$ within $10 \mathrm{~mm}$ in diameter. Luminance value of the improved LP model with white HP-LED was averaged $22,000 \mathrm{~cd} / \mathrm{m} 2$.

We thought that the luminance uniformity as luminance standard source in practice has been achieved. Then, NMIJ has the plan to use the standard LED for luminance developed in this study for disseminating of luminance scale.

\section{Acknowledgment}

This work was supported by JSPS KAKENHI Grant Number 15 K06126.

\section{References}

Suzuki, K., Kohmoto, K., et al. 2003. INTERNATIONAL ROUND ROBIN LED PHOTOMETRY TEST, Proc. of the 25th Session of the CIE, D2, pp.104 - 107.

GODO, K., SAITO, I., YAMAJI, Y., ISHIDA, K., WAKAKI, T. 2008. Development of high reliability LED transfer standard at NMIJ/NICHIA. Proc. of NEWRAD 2008, pp. 217 - 218.

GODO, K., ZAMA, T., YAMAJI, Y., ISHIDA, K., MATSUOKA, S. 2012. DEVELOPMENT OF A TRANSFER STANDARD FOR LUMINOUS FLUX MEASUREMENT OF HIGH POWER LEDS. Proc. of CIE 2012, pp. $619-622$.

NAKAZAWA, Y., GODO, K., NIWA, K., ZAMA, T., YAMAJI, Y., MATSUOKA, S. 2018. Development of LED-based standard source for total luminous flux calibration. Lighting Research \& Technology, https://doi.org/10.1177/1477153518800746. 
TAUBERT, D.R., HOLLANDTO, J., GUGG-HELMINGER, A. 2005. Compact Large-Area Uniform Color-Selectable Calibration Light Source, Proc of NEWRAD 2005, pp. 79 - 80.

FRED, M. 2014. Laser Beam Shaping- second edition - 1986, Publicaiton CRC Press.

VOELKEL, R.J., WEIBLE, K. 2008. Laser Beam Homogenizing: Limitations and Constraints, Proc. of SPIE - the International Society for Optical Engineering, 7102.

SALES, T.R.M. 2003. Structured microlens arrays for beam shaping, Opt.Eng.42, pp.3084-3085.

GODO, K. 2017. Investigation of new transfer standard for luminance by means of ray tracing simulation, Journal of Light and Visual Environment, 41, pp. 165 - 170.

Zemax $^{\mathrm{TM}}$ : http://www.zemax.com/ 